CGPG-98/11-2

\title{
The internal description of a causal set: What the universe looks like from the inside
}

\author{
Fotini Markopoulou \\ Center for Gravitational Physics and Geometry \\ Department of Physics \\ The Pennsylvania State University \\ University Park, PA, USA 16802 \\ fotini@phys.psu.edu
}

November 13, 1998

\begin{abstract}
We describe an algebraic way to code the causal information of a discrete spacetime. The causal set $\mathcal{C}$ is transformed to a description in terms of the causal pasts of the events in $\mathcal{C}$. This is done by an evolving set, a functor which to each event of $\mathcal{C}$ assigns its causal past. Evolving sets obey a Heyting algebra which is characterised by a non-standard notion of complement. Conclusions about the causal structure of the causal set can be drawn by calculating the complement of the evolving set. A causal quantum theory can be based on the quantum version of evolving sets, which we briefly discuss.
\end{abstract}




\section{Introduction}

In general, an entire spacetime, or an entire spatial slice in canonical gravity, can only be seen by an observer either in the infinite future or outside the universe. This is unphysical, so in the present paper we look for an alternative description of the causal structure of the spacetime that codes what an observer inside the universe can observe.

Another motivation for this work is to develop the proposal advocated in [目], that causality persists at the planck scale. Before one can argue whether or not this is plausible, there is the question of how to describe the causal structure. A spacetime metric is totally unsuitable since it is classical; when quantized it "fluctuates", leading to confusion in any argument, either for, or against, planckian causality.

An alternative to the Lorenzian metric which has been explored, for example, in [2] is a causal set. This is the discrete equivalent of a Lorentzian spacetime, the set of events in a discrete spacetime, partially ordered by the causal relations. Because the causal set can be defined prior to a spacetime manifold, it has been used in quantum gravity approaches that hold that a manifold is a classical concept, only found in a suitable classical limit of the planckian theory. Incorporating a causal set, as for example in [1, 4, 5], makes a theory "in principle" causal. However, the causal set itself is simply a very large collection of causal relations. If causality can instead be coded in an algebra, it may be possible to represent it directly in a quantum theory.

In order to address both of the above problems, we wish to introduce a transformation from the causal set $\mathcal{C}$ to the set of causal pasts of each event in $\mathcal{C}$. One possibility is to simply replace each event with its causal past. This has some advantages but it makes no progress towards a better coding of the causal structure. The maps between the causal pasts will be given exactly as in the causal set case. Therefore, we instead choose to work with evolving sets. An evolving set is a functor from the causal set to the category of sets. It is a generalization of an ordinary set that varies over the events in $\mathcal{C}$. It gives the causal past of each event but also contains the causal structure of $\mathcal{C}$ in an intrinsic fashion.

Evolving sets can be generalised to causal quantum theories, as we will discuss in this paper. Evolving sets satisfy a particular algebra, called a Heyting algebra, with operations which reflect the underlying causal set. In particular, the Heyting algebra complement is a measure of the non-triviality of 
the causal structure of $\mathcal{C}$. This algebra corresponds to a type of non-standard logic whose development, historically, is closely related to the passage of time.

In short, the evolving set of causal pasts contains the same information about the causal structure as the causal set, but it this information can be given in terms of algebraic relations. An algebraic way to express time evolution - as opposed to time given geometrically as an extra dimension - is likely to be advantageous in addressing quantum gravity issues.

In broader terms, the use of varying sets, of which the evolving ones which we study here are a particular case (those that vary over a causal set), applies to several occasions in physics when we need to make explicit the conditions under which a physical statement is made. In the present work, and generally in a causal quantum theory, we need to make explicit the time (event) dependence. Isham [6] and Isham and Butterfield [7] who introduced varying sets in physics have used them to specify consistent sets of histories and the different levels of coarse-graining.

The outline of the paper is the following. In section 2, we give the definition of a causal set and explain when we regard the causal structure of $\mathcal{C}$ to be trivial. In section 3 , we give a preview of the final construction of the past-sets over $\mathcal{C}$ to indicate what technical tools are needed and motivate some of the rather technical sections that follow. Since the evolving set of causal pasts is a generalisation of an ordinary set, in section t we review material from set theory. In particular, we examine the definition of a subset of some set in standard set theory. Then, we give the definition of a subset in categorial terms, since this is the form which we will generalise. The mathematical side of this material can be found in the standard mathematical literature on topos theory, for example in Mac Lane and Moerdijk [8], and we mainly concentrate on its physical interpretation.

In section 5, we construct the evolving set over a general causal set. In section 6, we apply this definition to the example of discrete Newtonian histories, a particularly simple case of a causal set. In section ฤ, we define the complement of a causal set and show that it is empty when the causal set is a lattice. The complement provides an algebraic definition of eventdependent causal horizons and we examine the possibility of generalising this to global properties of the causal set, such as black holes and branchings.

The complement is one of the operations of the Heyting algebra which the evolving sets obey. We give the definitions of all the four operations in a Heyting algebra in section 8, and we compare it to the standard Boolean 
algebra of ordinary sets.

Just as the boolean algebra of set theory implies an underlying boolean logic, the Heyting algebra of evolving sets means that the underlying logic is intuitionistic. For completeness, we translate the algebraic operations of Section 8 to logical ones in the Appendix. Finally, in section 9, we outline possible generalisations of the present results to causal spin networks and the quantum theory.

\section{Review of causal sets}

A causal set $\mathcal{C}$ is a discrete partially ordered set with structure that is intended to mirror that of Lorentzian spacetime. Namely, for any pair of points $p$ and $q$ either one is to the future of the other, say $p \leq q$, or they are causally unrelated. The ordering relation is antisymmetric, if $p \leq q$ and $q \leq p$, we must conclude that $p=q$ since timelike loops are not allowed. That the causal set is discrete means that the cardinality of the set $\{r \in \mathcal{C}: p \leq r \leq q\}$ for any pair $p \leq q$ is finite. We will also assume that the causal set has a finite number of elements.

Two elements $p$ and $q$ in the causal set have greatest lower bound (g.l.b.), $r$, when $r$ is an element in the causal set such that $r \leq p$ and $r \leq q$ and, for any other element $z, z \leq p$ and $z \leq q$ implies $z \leq r$. Similarly, $p$ and $q$ have a least upper bound (l.u.b.) when there is an element $t$ in the causal set such that $p \leq t$ and $q \leq t$, and if there is some element $z$ later than both $p$ and $q$, then it must also be later than $t$. The existence of a l.u.b. for two elements means that they will eventually meet at that common future event, while their g.l.b. is their last common past event.

A supremum for a partially ordered set, if it exists, is an element $s$ in the partially ordered set later than every other element, that is, $p \leq s$ for all $p \in \mathcal{C}$. Similarly, an infimum is an element $i$ in the partially ordered set before every other element, $i \leq p$ for all $p \in \mathcal{C}$. An infimum in the causal set means a single first event for the whole universe, while a supremum is the single final event that all others lead to.

A lattice is a partially ordered set with a g.l.b. and a l.u.b. for every pair of elements $(p, q)$ and a supremum and infimum. We are mainly interested in causal sets with a finite number of events. In this case, if there is a g.l.b. and a l.u.b. for each pair of events in the causal set, there is also a supremum 
and an infimum. When a causal set is a lattice, we will say that it has trivial causal structure. The reason for this is explained in section 7 .

We use the causal set to express time-dependence. The "position" of some event in the causal set is determined by its causal relations to the rest of the events in the causal set. To emphasize the fact that we use an event $p$ to specify a time instant relative to the rest of $\mathcal{C}$, we will often call it the stage $p$.

\section{The evolving set of causal pasts: the basic idea}

A classical observer at a given time instant can be placed on the corresponding event $p$ in the causal set without affecting the causal set. He knows about all the events in the past of $p$. In this minimal description, time passes when the observer moves to a later stage $q \geq p$ in the causal set. At $q$, the set of events in his causal past is larger but still includes all the past events of $p$.

If we are "outside" the causal set, what we see is a collection of such causal pasts, one for each event in $\mathcal{C}$. It is the thesis of this paper, however, that being outside the causal set is unphysical. We instead care about the same situation as viewed from "inside" $\mathcal{C}$ by one of the above observers. We want to know in what way the inside viewpoint is different from the outside one and if it has some interesting structure.

We will show that the inside viewpoint is given by a functor from the causal set category to the category of sets. Upgrading the causal set to a category involves nothing more than calling the events "objects" and the ordering relation $p \leq q$, when it exists, an "arrow $p \rightarrow q$ ". A collection of objects and arrows forms a category when the composition of the arrows is associative and there is an identity arrow. This is certainly the case for the causal set. From $p \leq q \leq r \leq s$ we can conclude that $p \leq s$ independently of the order in which we removed the mid-events (associativity) and $p \leq p$ for any $p \in \mathcal{C}$ (identity).

The category of sets, Set, which we need for the sets of causal pasts, has

sets for its objects and functions between sets for arrows. Composition of functions is associative, $(f \circ g) \circ h=f \circ(g \circ h)$, and for each set there is an identity map from the set to itself. 
Our task is the following: we need to go from the causal set $\mathcal{C}$ to the events in the past of each $p \in \mathcal{C}$. The former belong to the causal set, while the latter are objects in Set. One can go from one category to another, while preserving the structure of the first, by a functor. So, we employ the functor Past from the causal set to the category of sets,

$$
\text { Past }: \mathcal{C} \rightarrow \text { Set. }
$$

It has components $\operatorname{Past}(p)$ for each $p \in \mathcal{C}$ which are the events that lie in the past of that $p$,

$$
\operatorname{Past}(p)=\{r \in \mathcal{C}: r \leq p\} .
$$

They are sets. That Past is a functor means not only that it spits out Past $(p)$ for each $p$ but also that whenever $p \leq q$ it gives

$$
\operatorname{Past}_{p q}: \operatorname{Past}(p) \rightarrow \operatorname{Past}(q)
$$

which takes the causal past of $p$ to the causal past of $q$. Past preserves composition,

$$
\text { Past }_{p r}=\text { Past }_{q r} \circ \text { Past }_{p q} \quad \text { whenever } \quad p \leq q \leq r,
$$

and Past $_{p p}$ is the identity map,

$$
\text { Past }_{p p}: \operatorname{Past}(p) \rightarrow \operatorname{Past}(p) .
$$

Clearly, when $p \leq q$, the past of $q$ contains the past of $p$ and the map Past $_{p q}$ of equation (3) is really just set inclusion,

$$
\operatorname{Past}_{p q}: \operatorname{Past}(p) \subseteq \operatorname{Past}(q) .
$$

(The reason we bother with properties (4) and (5) which are obvious when Past $_{p q}$ is set inclusion is that we are interested in later generalising the functor Past and assign, for example, open spin network states to each $p$, or turn Past $_{p q}$ into a dynamical evolution arrow.)

The main role of Past is to transform events in $\mathcal{C}$ into sets (of events). In fact, functors from a causal set to Set, like Past, themselves form a category. These functors are not far from being sets, which is why they can be thought of as evolving, or varying sets, a generalisation of ordinary sets which can be found, for example, in [8]. We will, therefore, understand Past by generalising standard set theory. We start by giving a review of the relevant material for sets. 


\section{Sets and subsets}

We start with a review of material from set theory. Consider a set $X$ and a subset of it, $A$. How do we list those elements $x \in X$ which are also contained in the subset $A$ ? We use the characteristic function for $A$,

$$
\chi_{A}: X \rightarrow\{1,0\}
$$

defined by

$$
\chi_{A}(x)= \begin{cases}1 & \text { if } x \in A \\ 0 & \text { otherwise }\end{cases}
$$

or, diagramatically,

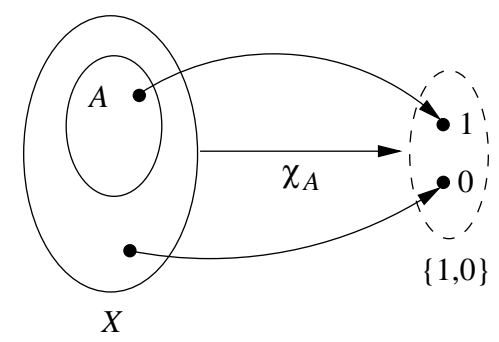

The function $\chi_{A}$ splits the elements of $X$ into those that also belong to $A$ (which get the label 1) and those that do not (which are marked 0). The subset $A$ can be recovered using the inverse of the characteristic function, as the inverse image

$$
A=\chi_{A}^{-1}(1) .
$$

What we have in mind for our time-dependent setup is a generalised notion of a subset, appropriate for evolving sets. In order to generalise, let us first scrutinize the above construction. Let us also treat sets as objects in the category Set, since we will later have to use evolving sets as objects in the apropriate category.

As we have already said above, the category Set has sets as its objects and its arrows are set functions. Of course, sets contain elements, and this is essential in the above definition of a subset. On the other hand, when we lay out the category Set there is nothing about elements to start with. How do they come about?

Set is a category with a terminal object. A terminal object in a category has the property that there is a unique arrow from any other object in the 
category to it. If it exists, the terminal object is unique up to isomorphism.t In Set, the terminal object should then be a set that is special in that there is a unique function from every other set into it. We can check that this is the one-element set in the following way. The function $g(x):=$ constant on an element $x \in X$, exists for any set $X$. Since its only output is constant, for each $X$ the function $g$ is unique (up to isomorphism). Thus, the terminal object is the set with the single element constant. We will denote the terminal object $\{0\}$.

Note that we only care about the fact that the terminal object has only one element and not about which one this element is since all one-element sets are isomorphic and our constructions only need to be good up to isomorphism. We therefore denote it by $\{0\}$ but we could just as well have used $\{1\},\{e\}$ or $\{$ constant $\}$ (and the 0 in the terminal object has absolutely nothing to do with the 0 in the set $\{1,0\})$.

Even if we are not told anything about the objects of Set having elements, we can infer that they do from the existence of a terminal object. Consider a function from $\{0\}$ to some set $X$. Since the range of this function is a single element, the only output it can have is an element of $X$. Thus, the functions from $\{0\}$ to $X$ are in one-to-one correspondence with the elements of $X$, and we can, in fact, define an element of $X$ to be such a function.

This may seem a far too complicated way of doing things, and it certainly is if all we cared about were sets. We need the concept of elements since we need to tell which events in $\mathcal{C}$ have occured at any given time. The above definition of an element proves useful in the rest of the present work because it can be generalised to evolving sets.

Going back to the subset $A$, we have

$$
A \stackrel{\subseteq}{\longrightarrow} X \stackrel{\chi_{A}}{\longrightarrow}\{1,0\}
$$

The first thing to note is that when we refer to $A$ as the subset of $X$ we mean that there is an inclusion function from $A$ to $X$. To emphasize this, we will give the name $f$ to this function,

$$
A \stackrel{f}{\longrightarrow} X \stackrel{\chi_{A}}{\longrightarrow}\{1,0\} .
$$

\footnotetext{
${ }^{1}$ In Set there is also an initial object, a set that is special in that there is a unique arrow from it to every other set in the category. With some thought we can convince ourselves that this is the empty set.
} 
The characteristic function $\chi_{A}$ is an equivalent description of the function $f$.

We choose to interpret 1 as "it is true that $x$ is in $A$ ", i.e. 1 is chosen as the true one of the two truth-values of the set $\{1,0\}$. [f "Choosing the element 1 " from $\{1,0\}$ as described above, means that we use a function from the terminal object to the set $\{1,0\}$ that outputs the element 1 ,

$$
T:\{0\} \longrightarrow\{1,0\} \quad \text { such that } \quad T(0)=1 .
$$

Its name $T$ is suggestive of "true", since this function represents our choice for which element of $\{1,0\}$ makes the expression " $x \in A$ " be true.

In its full detail then, the set inclusion diagram (11) is therefore

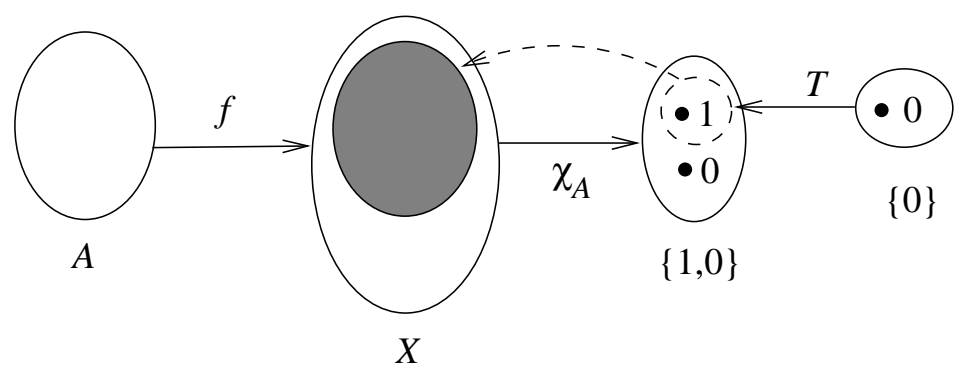

The shaded set is the image of $f$ of $A$ in $X$, or, equivalently, the inverse image $\chi_{A}^{-1}(1)$ in $X$.

\section{A subset is a pullback}

What the characteristic function $\chi_{A}$ does is pick the elements of $X$ that carry the label 1 from the two possible in $\{1,0\}$. The subset $A$ is then defined to be the set that contains those elements only.

The philosophy here can be roughly interpreted as follows. The set of truth-values $\{1,0\}$ is the simplest set we have that, together with the simplest possible (but non-trivial) inclusion function $T:\{0\} \rightarrow\{1,0\}$, exhibits all the features of a subset of a set. The characteristic function $\chi_{A}$ "lifts off" this model case of a subset, applies it to the particular set $X$ we provide, and returns the subset $A$.

\footnotetext{
${ }^{2}$ We are effectively replacing the set $\{1,0\}$ by the set $\{$ true, false $\}$ of boolean truth values.
} 
The mathematical explanation of the same philosophy is a pullback diagram. We group the functions $f: A \rightarrow X$ of eq. (11), $\chi_{A}: X \rightarrow\{1,0\}$ of eq. (7) and $T:\{0\} \rightarrow\{1,0\}$ of equation (12) into the diagram

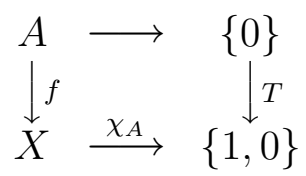

where the top arrow is the unique function from $A$ to $\{0\}$ (the function $g$ we defined above).

We know that $A$ contains those $x \in X$ with $\chi_{A}(x)=1$. This is precisely the statement that the diagram above is a pullback square, i.e. that $A$ is the set that contains $x \in X$ that have the same image under $\chi_{A}$ as $T(0)$,

$$
\chi_{A}(x)=1=T(0) .
$$

We say that $A$ is the pullback in the above diagram. In terms of functions, $\chi_{A}$ is the only function from $X$ to $\{1,0\}$ along which the true function $T$ pulls back to yield the inclusion function $f$, i.e. $f$ is the pullback of $T$ along $\chi_{A} \cdot$.

At this stage we are done with all the technical material from set theory. We will now go ahead to apply it, generalise it, and draw conclusions from it. The generalisation will attempt to capture the following: In the case of sets and subsets that we have seen, it is characteristic that $\chi_{A}$ splits the original set in exactly two parts, those that belong to the subset and those that do not, with anything in the middle excluded. In such cases we only need two truth-values and $\{1,0\}$ suffices. For example, this defines the causal past of a single event in the causal set: we can assign 1 to all events $r \leq p$ and 0 to all $q \geq p$.

However, we aim for more. We wish to have truth-values that inform us, not only whether an event has occured or not, but also how long we need to wait till it does, or, whether it will not happen no matter how long we wait. This is provided by the enlarged set of truth-values (in fact a functor) and the characteristic function for the evolving set Past.

\footnotetext{
${ }^{3} \mathrm{~A}$ subset is a special case of a pullback. In general, that $A$ is a pullback means it contains pairs of elements $(x, 0)$ with $x \in X$ and $0 \in\{0\}$ that have the same image in $\{1,0\}$. It is because $A$ is a subset that the second argument in this pair is the single element 0 of the terminal object and, since we can unambiguously drop it, we think of $A$ as containing elements of $X$ only.
} 


\section{The evolving set Past}

The "viewpoint" of an event $p$ in the causal set, the history of the world to the knowledge of $p$, is the set of events in the causal set in the past of $p$. This is a subset of the whole causal set $\mathcal{C}$. Here we consider the causal pasts evolving over the causal set.

As we said in section 3 , pasts of events in $\mathcal{C}$ are the outputs of a functor from the causal set to sets,

$$
\text { Past }: \mathcal{C} \longrightarrow \text { Set }
$$

that assigns to each $p \in \mathcal{C}$ its past

$$
\operatorname{Past}(p)=\{r \in \mathcal{C}: r \leq p\}
$$

and to each causal relation $p \rightarrow q$, when it appears in the causal set, a function

$$
\operatorname{Past}_{p q}: \operatorname{Past}(p) \longrightarrow \operatorname{Past}(q)
$$

which includes the idenity map $\operatorname{Past}_{p p}: \operatorname{Past}(p) \rightarrow \operatorname{Past}(p)$.

As an example, here is a causal set
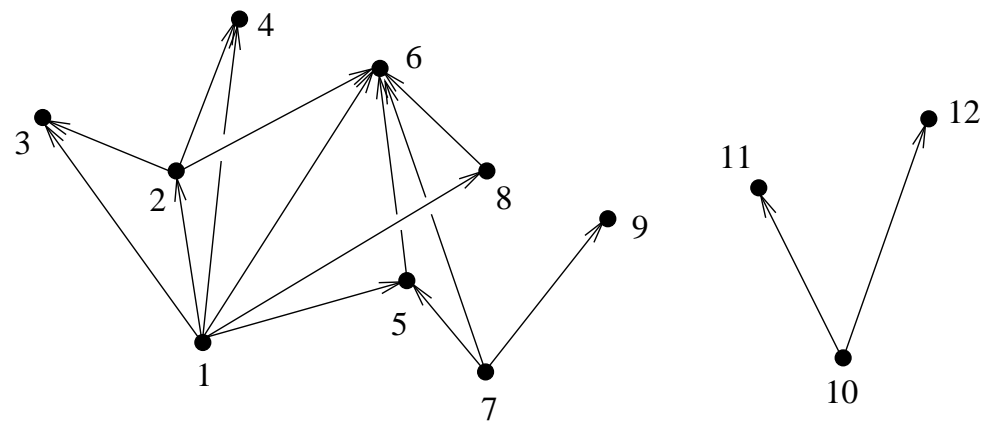

where we have drawn all the causal relations (and not only the nearest-event ones as is often done). When Past varies over this causal set, it gives the sets 
of causal pasts
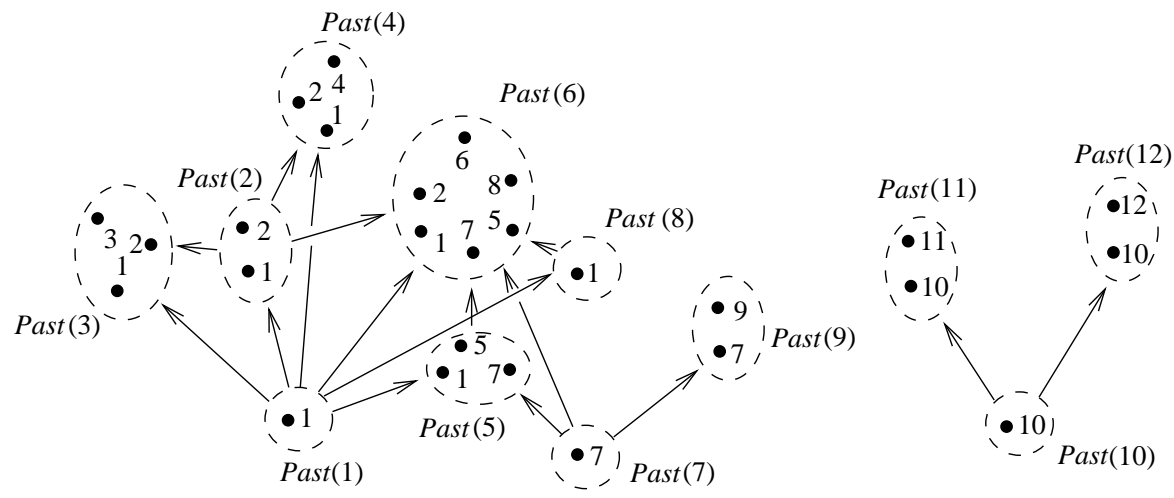

which themselves form a partially ordered set as the arrows are set inclusions.

Past is a $\mathcal{C} \rightarrow$ Set functor. It is a particular object in the category of functors from a partially ordered set $\mathcal{C}$ to the category of sets Set, denoted by Set $^{\mathcal{C}}$. (Arrows between these functors are natural transformations, an example of which we define below.)

Such functors, since they are evolving sets, are generalisations of sets. For this reason, the category $\mathbf{S e t}^{\mathcal{C}}$ has common features with the category Set of sets. It, also, has a terminal object, and it is a generalisation -in fact, an evolving version - of the one-element set that is the terminal object for sets. It is the functor t.o. which assigns to each $p \in \mathcal{C}$ the one-element set $\{0\}$

$$
\text { t.o. : } \mathcal{C} \longrightarrow \text { Set with t.o. }(p)=\{0\} .
$$

with an identity arrow between one-element sets for every existing causal link,

$$
\text { t.o.pq }:\{0\} \longrightarrow\{0\} \quad \text { when } \quad p \leq q .
$$

We can visualise the terminal object as the same web of relations as in $\mathcal{C}$, but with $\{0\}$ stuck in place of every event:

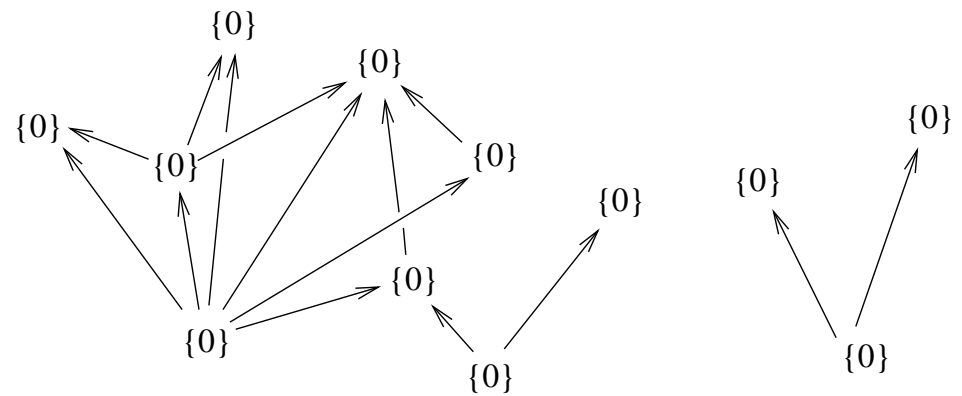


What the causal set encodes is whether one event is before or after some other event. Let us examine this. Suppose we are at event $p$ and need to say whether some other event $r \in \mathcal{C}$ has occured. Clearly, if $r \leq p$, then $r$ has happened. We can, however, do better than this and assign truth-values at $p$, not only to " $r$ precedes $p$ ", but to when $r$ will happen. Suppose there is some $q$ in the future of $p, q \geq p$, for which $r$ is past, $r \leq q$. At $p$, the "time" we need to wait before $r$ occurs is the causal relation $p \rightarrow q$. 1 This arrow is therefore a candidate for the truth-value, at $p$, for $r$ to have occured.

Under more careful inspection, we can see that a single arrow is insufficient. First, if $q$ has both $p$ and $r$ in its past, so will an event $q^{\prime} \geq q$. That is, the causal relations $p \rightarrow q^{\prime}$ for all $q^{\prime} \geq q$ should also be included in the truth-value we are calculating. Second, in general, there will not be a single first event $q$ in the common future of $p$ and $r$, but a set of such events, as is the case in the diagram below. These, and all events in their future, should be the causal relations in the truth-value at $p$ for $r$ having occured. They are the bold arrows in the following diagram.

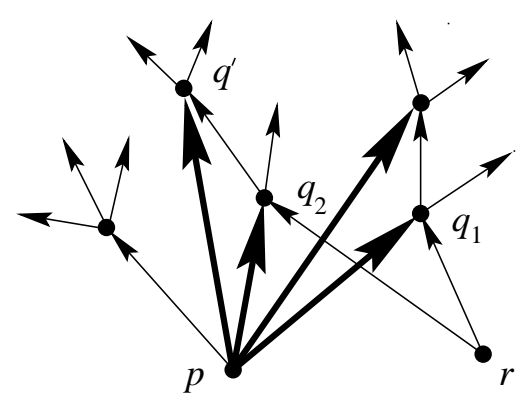

Let us formalize this. Let us define $R(p)$ as the set of all causal relationships that start at $p$ (including $p \leq p$ ),

$$
R(p)=\left\{\text { all } p \longrightarrow p^{\prime}: p \leq p^{\prime} \text { in } \mathcal{C}\right\} .
$$

Next, we define subsets $S(p)$ of $R(p)$ such that, if $S(p)$ contains the causal relation $p \rightarrow q$, it should also contain $p \rightarrow q^{\prime}$, for every $q^{\prime} \geq q$. $S(p)$ is

\footnotetext{
${ }^{4}$ An example of "length of time" truth-values is Newtonian histories, which we work out in the next section. There, the arrow $p \rightarrow q$ can be assigned a length, the number of events between $p$ and $q$. This, of course, is only possible because in the Newtonian case the causal set is a full linear order. In general, it is the arrow itself that we think of as the "time elapsed" between $p$ and $q$.
} 
therefore a left-extendible subset of $R(p)$ since the above definition can also be read as, if $(p \rightarrow q) \in S(p)$, and there is $\left(q \rightarrow q^{\prime}\right) \in \mathcal{C}$, then $\left(p \rightarrow q^{\prime}\right)=$ $\left(q \rightarrow q^{\prime}\right) \circ(p \rightarrow q) \in S(p)$. Namely, $S(p)$ is closed under left multiplication (extending a causal relation to the future).

A left-extendible subset $S(p)$ of $R(p)$ (in our conventionf) is called a sieve on $p$. The truth-value at $p$ that we are looking for is precisely a sieve. In fact, since it is not simply a yes/no truth-value, but also tells us at $p$ when $r$ will occur, it is called a time-till-truth value. A sieve is the type of truth-value appropriate to statements which, once they become true, they remain true, as is the case for an event having occured.

Therefore, at $p$, we have a sieve truth-value for every other event $r$ in the causal set. The set of all these sieves is the set of truth-values at $p$. We call this set $\Omega(p)$,

$$
\Omega(p)=\{\text { sieves on } p\} .
$$

A special subset of the events in $\mathcal{C}$ is, of course, those in Past $(p)$. There is a sieve in $\Omega(p)$ that corresponds to these particular events. They are special because at $p$ they have already happened. While we may think of the sieve truth-value in $\Omega(p)$ for $r \notin \operatorname{Past}(p)$ as a partially true value, the sieve for the events in $\operatorname{Past}(p)$ is the totally true value. We can use this understanding to find the sieve in $\Omega(p)$ that corresponds to the events in $\operatorname{Past}(p)$ and thus the set $\operatorname{Past}(p)$ itself. Since these events have occured at $p$ and at all events that follow $p$, the truth-value we are looking for is the entire $R(p)$, the maximal sieve in $\Omega(p)$.

In more detail, $R(p)$ is an element of $\Omega(p)$ which is selected in the manner described in section 4 for choosing elements of a set using the terminal object. We define an inclusion of $\{0\}$ into $\Omega(p)$, which we call the totally true arrow at $p, T_{p}$ :

$$
T_{p}:\{0\} \longrightarrow \Omega(p)
$$

such that

$$
T_{p}(0)=R(p)=(\text { maximal sieve on } p) .
$$

Let us now observe that $\operatorname{Past}(p) \subset \mathcal{C}$ and call this inclusion $f_{p}$,

$$
f_{p}: \operatorname{Past}(p) \subseteq \mathcal{C}
$$

\footnotetext{
${ }^{5}$ We call a sieve what in $[8]$ is a cosieve.
} 
Both $\operatorname{Past}(p)$ and $\mathcal{C}$ are sets, and as we saw in section 2, that the former is a subset of the latter means that $\operatorname{Past}(p)$ is the pullback in the diagram

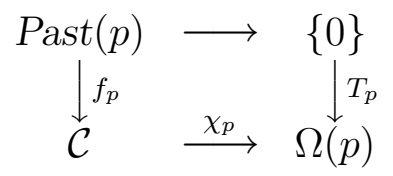

namely, $\operatorname{Past}(p)$ contains those events $r \in \mathcal{C}$ that satisfy

$$
\chi_{p}(r)=(\text { maximal sieve on } p)=T_{p}(0),
$$

exactly as we saw in section 4 .

We have, therefore, found that the set of truth-values $\Omega(p)$ at a given event $p$ is the set of sieves on $p$ and, in particular, that the maximal sieve gives the component Past $(p)$ of Past at $p$. We next have to let these results "vary" over $\mathcal{C}$ so that we can define the functor Past by a generalisation of what we did for $\operatorname{Past}(p)$.

As we move from $\operatorname{Past}(p)$ to $\operatorname{Past}(q)$, the appropriate truth-values are consistently given by $\Omega(p)$ and $\Omega(q)$, so that we can find $\operatorname{Past}(q)$ given $\operatorname{Past}(p), p \leq q$ and $\mathcal{C}$. That is, the diagram

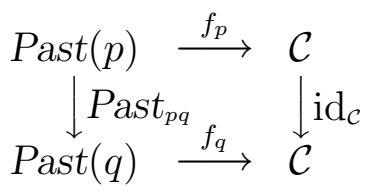

commutes $\left(\operatorname{id}_{\mathcal{C}}\right.$ is the identity operation on the causal set). On the left of the diagram we have components of the functor Past. We can organize the righthand side in the same way by constructing the constant functor

$$
\text { World }: \mathcal{C} \longrightarrow \text { Set }
$$

with

$$
\begin{aligned}
\operatorname{World}(p) & =\mathcal{C} \quad \text { for any } p \in \mathcal{C}, \\
\operatorname{World}_{p q} & =\operatorname{id}_{\mathcal{C}} .
\end{aligned}
$$

Now the diagram (31) reads

$$
\begin{array}{ccc}
\operatorname{Past}(p) \stackrel{f_{p}}{\longrightarrow} & \text { World }(p) \\
\downarrow \text { Past }_{p q} & & \downarrow \text { World }_{p q} \\
\operatorname{Past}(q) \stackrel{f_{q}}{\longrightarrow} & \operatorname{World}(q)
\end{array}
$$


What this diagram says is that Past is a subfunctor of World, i.e. there is an inclusion

$$
f: \text { Past } \longrightarrow \text { World, }
$$

which is a natural transformation, i.e. it has components $f_{p}, f_{q}$ that make the diagram (35) commute, $f_{q} \cdot$ Past $_{p q}=\operatorname{World}_{p q} \cdot f_{p}$ for every two events $p, q$ that are causally related.

The set of truth-values for $\operatorname{Past}(p)$ is $\Omega(p)$ while for Past $(q)$ it is $\Omega(q)$. These, too, are components of the functor

$$
\Omega: \mathcal{C} \longrightarrow \text { Set }
$$

that for each event in $\mathcal{C}$ outputs its set of truth-values, the sieves on that event, and has functions $\Omega_{p q}: \Omega(p) \rightarrow \Omega(q)$ that, given the set of sieves on $p$ give the set of sieves at $q$.

We, therefore, have a characteristic function $\chi$ from World to $\Omega$,

$$
\chi: \text { World } \longrightarrow \Omega \text {. }
$$

which is also a natural transformation, it has components at each event that compose as $f$ did above. We obtain the particular subfunctor Past, when, at each event in the causal set, $\chi$ maps into the maximal sieve at that event. Namely, the function $T_{p}$ above is the $p$-component of the arrow $T$ from the terminal object t.o. for $\mathbf{S e t}^{\mathcal{C}}$ defined in (20) to $\Omega$,

$$
\begin{array}{cll}
T & : & \text { t.o. } \longrightarrow \Omega, \quad \text { with } \\
T_{p} & : & \text { t.o. }(p)=\{0\} \rightarrow \Omega(p) .
\end{array}
$$

We, finally, have everything we need to give the subfunctor Past as a pullback. All four Past, World, t.o. and $\Omega$ are objects in the category Set $^{\mathcal{C}}$. We may then construct the diagram

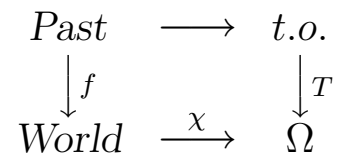

and ask that the functor Past makes this diagram pullback. This means that it reduces to the diagram (31) consistently at each event in the causal set. 
Past is a subfunctor of World.

We now know how to specify an evolving set. We have defined an evolving causal past by giving the functor Past that generates the causal past of each event and corresponding evolving set of truth-values $\Omega$. We can now analyze their physical significance.

Overall, we have achieved two things. One is to express the causal structure in terms of causal pasts at each event, which we regard as physically more satisfactory than an entire spacetime or causal set. This is the description that can be given from an observer inside the causal set.

Second, even when the causal set is not a lattice, the evolving sets over it is one. As we will see next, they satisfy a particular algebra, called a Heyting algebra, whose operations reflect the underlying causal set. Thus, we can give the causal structure algebraically. We do this in the section 8 .

Before we continue with the algebra of causal sets, we will give a simple example of causal pasts in a causal set. We will consider histories in a discrete Newtonian universe.

\section{Discrete Newtonian histories}

Discrete Newtonian time evolution is the case where integers $0,1,2,3, \ldots \in \mathbf{N}$ can label the preferred time parameter. In a Newtonian world, we have a preferred foliation with slices $S_{0}, S_{1}, S_{2}, S_{3}, \ldots$ labelled by the time stage when they occur. It is, therefore, a very special causal set: the fully ordered set of integers.

At some given time $n$, we may ask for the history up to that time. It is the sequence of slices up to $n$, namely,

\footnotetext{
${ }^{6}$ All of Past, t.o., World and $\Omega$ are evolving sets, and $\chi, T$ and $f$ are natural transformations. In particular, $\chi$ has components

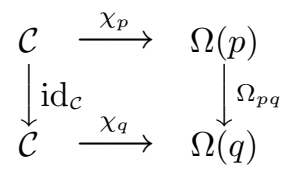

${ }^{7}$ In Newtonian evolution the speed of light is infinite and we do not have the local (bubble) evolution present in a causal universe. Effectively, the slice $S_{n}$ can simply be replaced by $n$, or, equivalently, we can reduce the slice to contain a single event and the causal
} 


$$
\operatorname{History}(n)=\left\{S_{0}, S_{1}, S_{2}, \ldots, S_{n}\right\} .
$$

Clearly there is a history at each time stage $n \in \mathbf{N}$. We can set up a functor History,

$$
\text { History }: \mathbf{N} \longrightarrow \text { Set, }
$$

which, when fed the time instant, spits out the history up to that time, History $(n)$, as given by (43). The functor History also provides maps between histories which are just the inclusions of the shorter histories into the longer ones,

$$
\operatorname{History}_{n m}: \operatorname{History}(n) \subseteq \operatorname{History}(m) \quad \text { whenever } n \leq m \text {. }
$$

We may now want to ask whether some slice $S_{i}$ is already in $\operatorname{History}(n)$ and, if not, when it will be included. First, we note that all slices can be found in the components of the constant functor

$$
\text { World : } \mathbf{N} \longrightarrow \text { Set, }
$$

since its components are

$$
\operatorname{World}(n)=\left\{S_{0}, S_{1}, S_{2}, \ldots\right\} \quad \text { for any } n \in \mathbf{N} \text {. }
$$

History is a subfunctor of World since, at each time $n, \operatorname{History}(n) \subseteq$ $W \operatorname{orld}(n)$,

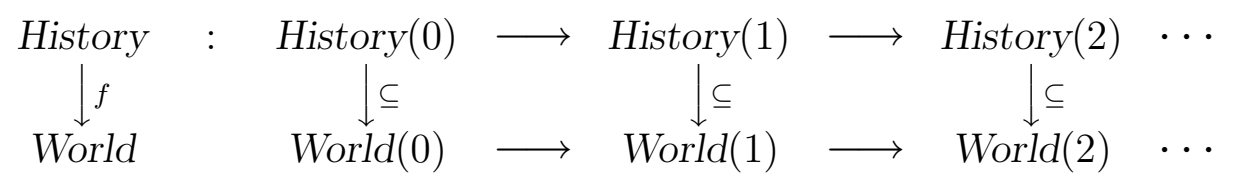

(By analogy to the section on sets, World is the evolving set version of the set $X$, while History is an "evolving subset" in place of $A$.)

structure will not change. Therefore, the discrete Newtonian history $\left\{S_{0}, S_{1}, S_{2}, \ldots, S_{n}\right\}$ is isomorphic to just $\{0,1,2, \ldots n\}$. 
Diagramatically, we have

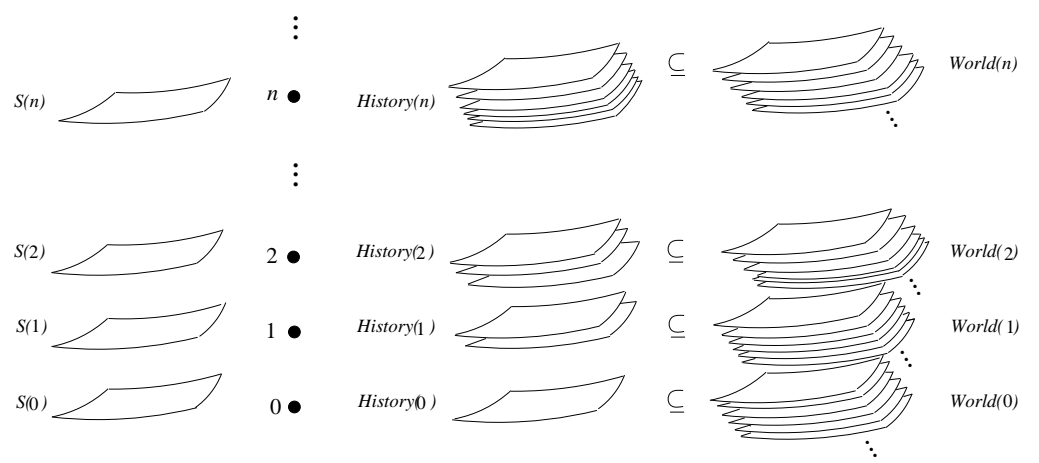

At time $n$, the slice $S_{i}$ may or may not be in $\operatorname{History}(n)$. If it is not already history at $n$, then, if we wait for some time $t$ we may find that $S_{i} \in \operatorname{History}(n+t)$. As a result, the characteristic function that tells us at $n$ when $S_{i}$ is history is

$$
\chi_{n}\left(S_{i}\right)= \begin{cases}\text { least } t & \text { at which } S_{i} \in \operatorname{History}(n+t), \text { if this occurs } \\ \infty & \text { otherwise. }\end{cases}
$$

Of the numbers that $\chi_{n}\left(S_{i}\right)$ spits out, 0 is to be understood as "true now" $\left(S_{i}\right.$ is already history at time $\left.n\right), 1$ is "true tomorrow" and so on. $\infty$ is the symbol we chose for "never true", if the particular slice $S_{i}$ never appears in History.

Note that $\chi_{n}$ takes its values in the set $\mathbf{N}_{\infty}$, the integers together with $\infty$. Therefore, the set of truth-values at $n$ is $\mathbf{N}_{\infty}$. In fact, the set of truth-values is $\mathbf{N}_{\infty}$ at any time. We can chain these sets together to form $\Omega: \mathbf{N} \longrightarrow$ Set, that represents the time-development of the set of truth-values, only, in our especially simple case, there is no time development and

$$
\Omega(n)=\mathbf{N}_{\infty} \quad \text { for any } n \in \mathbf{N} .
$$

$\Omega$ then is the "constant evolving set" (with identity maps between components)

$$
\Omega=\left\{\mathbf{N}_{\infty}, \mathbf{N}_{\infty}, \mathbf{N}_{\infty}, \mathbf{N}_{\infty}, \ldots\right\} .
$$

Note that the true now (at $n$ ) output of $\chi_{n}$ in (50) is the value 0 . This is the maximal sieve in $\Omega(n)$ above. We can see that this is because the sieves here tell us how long we need to wait before $S_{i}$ joins History, and 0 is, of 
course, the shortest possible wait. It is the maximal sieve since it contains all longer waits, $1,2, \ldots, n$, etc. P

Finally, the history at a given time can be obtained by first defining the function $T_{n}$ at $n$ to have output 0 which is the true now value in (50).

$$
T_{n}:\{0\} \rightarrow \Omega(n) \quad \text { with } \quad T_{n}(0)=0 .
$$

Then History $(n)$ is the pullback in

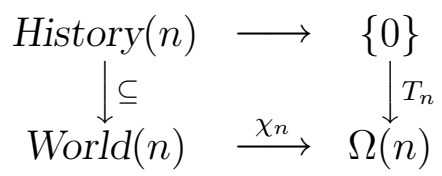

namely, it contains those slices $S_{i}$ in $\operatorname{World}(n)$ that satisfy

$$
\chi_{n}\left(S_{i}\right)=0=T_{n}(0),
$$

and thus are already true at $n$.

\section{$7 \quad$ The complement of Past measures the causal structure of $\mathcal{C}$}

In section 5 we provided a time-dependent way of telling when some event in a causal set has occured (equations (38) and (25)). What characterises an

${ }^{8}$ We can write $\Omega(n)$ as all the arrows from $n$ to any $m \geq n$, i.e. all the possible "waiting times", or time-till-truth values:

$$
\Omega(n)=\left\{\begin{array}{l}
n \longrightarrow n \\
n \longrightarrow n+1 \\
\vdots \\
n \longrightarrow n+k \\
n \longrightarrow n+k+1 \\
\vdots \\
n \longrightarrow \infty
\end{array}\right.
$$

The length of these arrows is the number that $\chi_{n}$ of eq. (50) outputs. These are the sieves on $n$. Going from $\Omega(n)$ to $\Omega(n+1)$, the length of each arrow goes down by one since, what at $n$ was "true tomorrow", at $n+1$ is "true now" and so on. Thus $\Omega$ is closed under making the arrow $n \rightarrow m$ even longer, which means that what is true now will remain true tomorrow. The arrow $n \rightarrow n$ is the shortest one, it means no wait, i.e. true now (at $\mathrm{n}$ ). 
event that will never happen? In this section we give the relevant definition which encodes much of the causal structure of $\mathcal{C}$. We then make some first remarks about the possibility of defining algebraically global properties of a causal set, such as black holes and branchings.

A sieve on $p \in \mathcal{C}$ is the time-till-truth value for some other event $r \in \mathcal{C}$ to happen. In the same way, if $r$ will never happen, then it should satisfy

$$
r \notin \operatorname{Past}(q) \quad \text { for any } q \geq p,
$$

since $r$ never happening for $p$ means that it will never be in the past of the future of $p$.

The set of events satisfying (57) is the evolving set complement of Past $(p)$, the set of events in the causal set $\mathcal{C}$ that are not in the past of the future of $p$ :

$$
\neg \operatorname{Past}(p)=\{r \in \mathcal{C}: r \notin \operatorname{Past}(q) \text { for any } q \geq p\} .
$$

As a first example, consider the Newtonian case. There the functor History plays the same role as Past, and thus

$$
\neg \operatorname{History}(n)=\left\{k \in \mathbf{N}: S_{k} \notin \operatorname{History}(m) \text { for any } m \geq n\right\} .
$$

But, since the causal ordering is a full linear ordering, any slice will at some point join History. Thus, the complement of History $(n)$ is empty.

It is generally the case with a causal set that is a lattice that the complement of Past is empty. This is because, by definition, when $\mathcal{C}$ is a lattice any two events $p$ and $r$ have a least upper bound which is an event with both $p$ and $r$ in its past. Thus, definition (58) returns the empty set. Note that this implies that $\neg$ Past cannot distinguish between different lattice causal sets.

Second, let us take the very small causal set in (18). It is not a lattice. There is no single "big bang" event and no single "final crunch". Consider the event labelled 7 and let us find the complement of Past(7). $\neg \operatorname{Past}(7)$ is all the events not in the past of the future of 7 , i.e. $3,4,10,11$ and 12 . We 
mark them white:
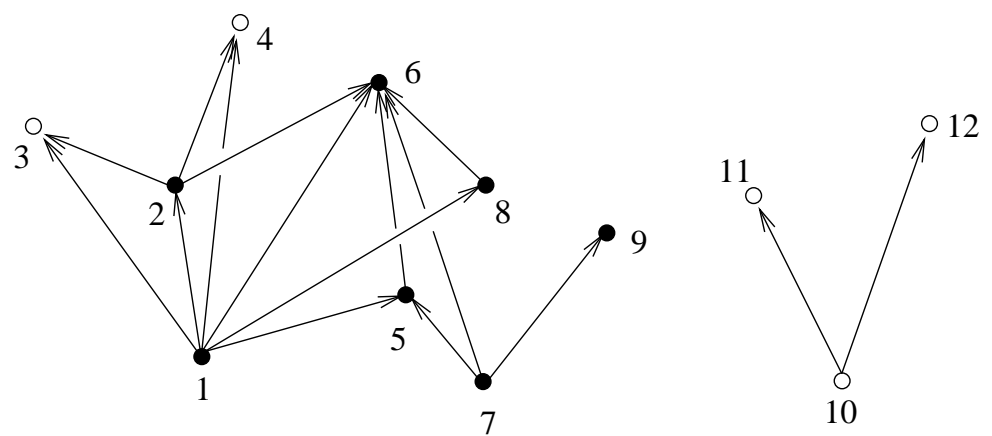

Let us imagine placing an observer on event 7. The future of 7 is 5,6 and 9. In these future times, he will receive information from anything in the past of 5,6 and 9, i.e. from 1,2, 5 and 8. He will never, however, be able to see $3,4,10,11$ and 12 , the events in $\neg \operatorname{Past}(7)$. As far as 7 is concerned, $\neg \operatorname{Past}(7)=\{3,4,10,11,12\}$ is beyond its causal horizon, 7 will never receive information from these events.

It is not suprising that there is such a boundary when the causal structure is nontrivial, and parts of the causal set are either disconnected or branch off. This is reminiscent of topology change in a spacetime, although, naturally, we do not have the same notion of topology here.

We should emphasize that this is a horizon for 7 and not a global property of $\mathcal{C}$. Such an event-dependent notion of causal horizon is useful in constraining a microscopic theory to reproduce the Einstein equations at large scale (used in [10]). However, since this paper is devoted simply to the exposition of the evolving set Past and not its applications, we will not go further into this, except for comments in the conclusion section.

A global property of the spacetime, such as a branching of the causal set or a black hole, needs, presumably, the agreement of the complements of a large class of events. A permanent braching of $\mathcal{C}$ will result to all events in one branch having (almost all) the events in the other branch in their complements. It is a very interesting but subtle problem to express a black hole in terms of the causal structure by considering intersections of the complements of the events in $\mathcal{C}$. This is work currently in progress.

The non-standard complement of Past is one of the four operations of the algebra of (evolving subsets of) evolving sets. It is a Heyting algebra, which we define in the next section by giving its four opreations. 


\section{Evolving sets obey a Heyting algebra}

Recall that Past, as a functor from $\mathcal{C}$ to sets, can be regarded as an evolving set, a set that varies over all events in $\mathcal{C}$. As with the other definitions in this paper, we start from sets and generalise to evolving sets.

\subsection{The algebra of sets}

Take a set $X$, and consider the set of its subsets, its powerset. There are four possible operations on the subsets of $X$, and they produce four unique new sets:

- Union: $(A \cup B)=\{x \in X: x \in A$ or $x \in B\}$.

- Intersection: $(A \cap B)=\{x \in X: x \in A$ and $x \in B\}$.

- Implication: $(A \Rightarrow B)=\{x \in X$ : if $x \in A$ then $x \in B\}=\{x \in X$ : $x \notin A$ or $x \in B\}$.

- Complement:

The complement $\neg A$ of $A$ is characterised by

$$
A \cap \neg A=\emptyset \quad \text { and } \quad A \cup \neg A=X,
$$

and, therefore, $\neg A=\{x \in X: x \notin A\}$.

Note that the complement can, in fact, be derived from the implication operation by defining

$$
\neg A=(A \Rightarrow \emptyset) .
$$

This means that $\neg A$ contains those $x \in X$ which "if they are in $A$ they also are in $\emptyset "$, which is a formal way to exclude the elements of $A$ from $\neg A$. As on other occasions in this paper, it is this twisted definition that we generalise below. From the two relations (61), one can prove the familiar

$$
\neg \neg A=A .
$$


Diagramatically, the four set operations are:
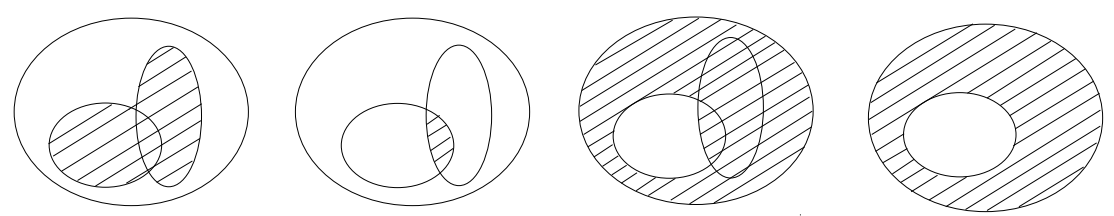

The partially ordered set of subsets of $X$ is a lattice, since every two subsets have their union as a l.u.b. and their intersection as a g.l.b. The infimum of the powerset of $X$ is the empty set $\emptyset$ and the supremum is $X$ itself. Importantly, it is a distributive lattice, i.e. the identity

$$
A \cap(B \cup C)=(A \cap B) \cup(A \cap C)
$$

holds.

A distributive lattice in which every element has a complement that satisfies (61) is a Boolean lattice, or, a Boolean algebra. Therefore, the subsets of $X$ form a Boolean algebra.

\subsection{The algebra of evolving sets}

With evolving sets, we have the same four operations of union, intersection, implication and complement, but different relations between them and thus a different algebra which is known as a Heyting algebra.

Consider two evolving sets, or functors from $\mathcal{C}$ to Set which are subfunctors of our constant functor World. Let us call them $F$ and $G$. We have worked with one of them, Past. Another example would be the functor generating events in the future of each event in $\mathcal{C}$, or sets of spacelike separated events in each causal past, etc.

The four possible operations on $F$ and $G$ are given componentwise (at each event in $\mathcal{C}$ ) but so that they preserve the causal structure of $\mathcal{C}$. They are: : $^{\text {ev }}$

\footnotetext{
${ }^{9}$ It is somewhat difficult to draw diagrams for evolving sets. If the reader wishes to have an illustration of a Heyting algebra, a good alternative is to draw open sets. Open subsets of a given (open) set $O$ also satisfy a Heyting algebra. Given an open set $U \subset O$,
} 
- Union: $(F \cup G)(p)=F(p) \cup G(p)$.

- Intersection: $(F \cap G)(p)=F(p) \cap G(p)$.

- Implication:

$$
\begin{aligned}
(F \Rightarrow G)(p)= & \{r \in \mathcal{C}: \text { for any causal relation } f: p \rightarrow q, \\
& \text { if } r \in F(q), \text { then } r \in G(q)\} .
\end{aligned}
$$

That is, $(F \Rightarrow G)(p)$ contains all those events, which, if by the time step $f: p \rightarrow q$ join $F$ at $q$, they also join $G$ at $q$. One can check that this is a $\mathbf{S e t}^{\mathcal{C}}$ functor.

- Complement:

Since we have already worked with the complement of Past, we will discuss the complement using this particular example rather than the abstract functor $F$. We will basically give the justification for the definition (58). The problem with applying the definition (61) of a boolean complement to Past is that it gives its complement as all $r \in \mathcal{C}$ that are not in $\operatorname{Past}(p)$, namely $(\mathcal{C}-\operatorname{Past}(p))$. But, since a causal past keeps getting larger with time, i.e. $\operatorname{Past}(p) \subseteq \operatorname{Past}(q)$ when $p \leq q$, this complement would get smaller in time, $(\mathcal{C}-\operatorname{Past}(p)) \supseteq(\mathcal{C}-\operatorname{Past}(q))$. This has the wrong behavior to be a functor like Past and hence we cannot get an algebra of evolving sets using this complement.

Let us instead define the complement by generalising (62). First we need an evolving version of $\emptyset$. This is the functor Empty that assigns to each $p \in \mathcal{C}$ the empty set (with the expected inbetween arrows). We then get

$$
\neg \text { Past }=(\text { Past } \Rightarrow \text { Empty }) .
$$

In components this gives

$$
\neg \operatorname{Past}(p):=\{r \in \mathcal{C}: \text { for any causal relation } f: p \rightarrow q \text { in } \mathcal{C},
$$

the set-theoretic complement of $U$ (i.e. the one satisfying the definition (61)), which we will call $U^{c}$ to avoid confusion, is a closed set. If we want an algebra of open sets, we need, instead of $U^{c}$, to use the interior of the set-theoretic complement of $U, \operatorname{Int}\left(U^{c}\right)$. But then, clearly, $U \cup \operatorname{Int}\left(U^{c}\right) \subset O$ since the closure of $U$ has been left out. Open sets are the standard example of a Heyting algebra in the literature. That they behave in the same way as evolving sets is not surprising, roughly speaking, they both involve an infinite sequence, the former of time stages to the future, the latter of points to the boundary. 


$$
\begin{aligned}
& f(r) \notin \operatorname{Past}(q)\} \\
=\quad & \{r \in \mathcal{C}: r \notin \operatorname{Past}(q) \text { for any } q \geq p\} .
\end{aligned}
$$

which is the definition (58). This is a $\mathbf{S e t}^{\mathcal{C}}$ functor and an appropriate complement for Past.

Generally, $\neg$ Past satisfies

$$
\text { Past } \cap \neg \text { Past }=\text { Empty, }
$$

but

$$
\text { Past } \cup \neg \text { Past } \subset \text { World. }
$$

(An example of this is the case of a lattice causal set where the righthand side of $(70)$ is just Past.) Because the Heyting algebra complement satisfies the weaker set of conditions (69) and (70), instead of (61), it is often called a pseudo-complement. We simply call it complement, or a Heyting algebra complement. For a Heyting algebra complement, it is not true anymore that $\neg \neg$ Past equals Past (again consider the example where $\mathcal{C}$ is a lattice). In general, for some evolving set, the double complement is larger than the original.

The subobjects of a $\mathbf{S e t}^{\mathcal{C}}$ object like World, therefore, form a lattice: there is union and intersection of any two, World is the supremum and Empty the infimum. This is independent of whether the causal set itself is a lattice. This is significant because a lattice can be used as an algebra and, at the same time, evolving sets still contain all the causal information in $\mathcal{C}$.

The lattice of evolving sets is a distributive one, since for any three subobjects $F, G$ and $H$ of World,

$$
F \cap(G \cup H)=(F \cap G) \cup(F \cap H)
$$

holds.

A lattice that is distributive and has implication defined for every pair of elements is called a Heyting algebra. It is weaker than the boolean algebra (it is a generalisation). For a Heyting algebra element (evolving set) F,

$$
\neg \neg F \supseteq F .
$$

(but one can check that $\neg \neg \neg F=\neg F$ ). If it is the case that $\neg F \cup F=$ World, then $\neg \neg F=F$ and the Heyting algebra reduces to a boolean one. 
Summarising, the algebra of the analogue of the powerset of an evolving set is a Heyting algebra. A key feature of a Heyting algebra is the complement. It is intimately tied to the causal structure and therefore has physical significance.

\section{Using evolving sets in a causal quantum theory}

The reason we carried out the analysis of this paper is that it may serve in obtaining a quantum theory of gravity. We are keeping as much of the causal structure of general relativity as it is possible without using metric manifolds. Clearly, we are restricted to very basic features of a spacetime, namely, the causal ordering of events. No further qualifications of these events have been specified. It is true that it is possible to recover the metric of a spacetime up to a conformal factor from the causal relations between all its events. It has been argued that in the discrete case, even this factor may be fixed by assigning spacetime volume to each event [11]. However, in the causal set as it is used here, no straightforward relationship between its events and those of a classical spacetime has been assumed. In this case, the causal structure is not all that is needed.

As it has been proposed in [1] and further elaborated in [4, 5, 12], extra spatial structure can be attached to a causal set in the form of a spin network, a graph with edges labelled by representations of $S U(2)$ [13]. Spin networks have already been part of a quantized gravity theory in loop quantum gravity [14]. On the other hand, in the causal spin network scheme, as in other applications of causal sets, a problem is how to represent the causal structure in a way that is useful in the quantum theory. We hope that the present algebraic formulation of causality will resolve this, at least by restricting the quantum theory to the functorial form that implies underlying causality, as we will outline below. The application of evolving sets to spin networks and generally a causal quantum theory is currently being constructed. Here we indicate how this application may be carried out. 


\subsection{Elementary causal quantum theory}

Standard quantum theory is unambiguously applicable under certain conditions: that the time is fixed and that the separation between the system in question and its environment is also fixed.T Quantum field theory is, in principle, more flexible, however, the single Hilbert space employed restricts its use to a semiclassical regime of quantum gravity.

Leaving aside for the time being the system/environment issue, and keeping the causal set as the time evolution record, we may propose removing the fixed-time condition by using a Hilbert space for each event in the causal set. For example, in the Newtonian case, this results in a sequence of Hilbert spaces which we may regard as the evolution of the quantum universe.

Now we note the following. The present work suggests that the Hilbert spaces at each event may be regarded as objects in the category of Hilbert spaces, Hilb (with linear transformations as the arrows). Then, the causal structure is preserved in the quantum theory if there is a functor from the causal set to Hilb, $Q: \mathcal{C} \rightarrow$ Hilb. Let us check if this is the case. For each event $p \in \mathcal{C}$, we have a Hilbert space $H(p)=Q(p)$. However, given a causal relation $p \leq q$ in $\mathcal{C}$, it is not, in general, the case that there is a linear map from $H(p)$ to $H(q)$ such that $H(p)$ is a linear subspace of $H(q)$. Therefore, the ordering of $\mathcal{C}$ is not preserved by the functor $Q$ into Hilb.

Some of the ways to circumvent this problem and maintain causality in the quantum theory that we are aware of, is to restrict the linear maps, evolve linear subspaces of the above Hilbert spaces (evolve the projection operators), and replace the Hilbert spaces with algebras of observables at each event (see 9.3).

\footnotetext{
${ }^{10}$ At the conceptual level, varying sets is the method proposed by Isham [6 to accomodate contextual physical statements, where context means the conditions under which a certain physical description and physical statement is applicable. We have dealt with evolving sets (and in this section we discuss what may be called "evolving algebras" and "evolving state spaces") where the context is causality, namely we need to specify the event $p$ in order to get its classical past or the quantum theory at $p$. The same method can be used in a third context, to treat the system-environment split implied by standard quantum theory. Here the context is all possible systems in the universe. This is work in progress with C. Rovelli.
} 


\subsection{Causal spin networks}

Causal evolution of spin networks is in principle similar to the above scheme, but with the important difference that it leads to entangled states rather than states in a single Hilbert space (one Hilbert space for each event). Analyzing a functor from the causal set to entangled states or density matrices is beyond the scope of this paper, but we will illustrate how they arise.

One may regard a causal history of spin networks as a sequence of spin network graphs such that the nodes of all the graphs in a given history are the elements of the causal set and therefore are partially ordered."' Between the nodes of a single spin network, therefore, there are no causal relations. Assuming a causal history with connected graphs, the set of nodes of a spin network is maximal, i.e. there is not an element in $\mathcal{C}$ outside this set that is not causally related to some element in the set. A maximal set of causally unrelated events in $\mathcal{C}$ is a maximal antichain.

The complaint with which we started this paper, that a spatial slice can only be seen by an observer in the infinite future, is also present in the spin network evolution since an antichain requires the same observer. What this work suggests is that we should instead work with (partial) antichains at event $p$, for each $p \in \mathcal{C}$ ( $p$ is also a spin network node). These are sets of nodes in a causal spin network history that are causally unrelated inside $\operatorname{Past}(p)$.

Given the functor Past, we can construct the functor Antichains : $\mathcal{C} \rightarrow$ Set,

Antichains $(p)=\{$ sets of causally unrelated events in Past $(p)\}$.

For every causal relation $p \leq q$, the antichains at $p$ are, of course, a subset of those in $q$.

Given an element in Antichains $(p)$, there is a number of graphs that has these events as its nodes, i.e., for each $p$ we have the set $\operatorname{Graphs}(p)$ of all graphs with elements of Antichains $(p)$ as their sets of nodes. (We ignore the $S U(2)$ labels which further enlarge this set.) Clearly, Graphs $(p)$ includes open spin networks.

\footnotetext{
${ }^{11}$ In fact, it is the local changes of graphs which generate the history that are partially ordered into a causal set. The difference does not affect the present discussion. For more detail on this, see [5].
} 
An open spin network has free edges, all labelled by $S U(2)$ representations, and thus can be regarded as an entangled state. Hence, to go from open spin networks at $p$ to open spin networks at a later $q$, we need an evolution operator on density matrices.

\subsection{Observable causal quantum theory}

A further possibility is to replace the Hilbert spaces of the scheme in 9.1 with the algebra of observables at each event. $\mathrm{\square}$ This means using the functor

$$
O Q: \mathcal{C} \longrightarrow \mathcal{A}
$$

where $\mathcal{A}(p)$ will be the algebra of observables at $p$.

This scheme provides what we may regard as a quantum field theory on a causal set.

Concluding this section, we note that the use of functors from the causal set to our preferred description of the universe is a safe way to check that this description is indeed causal. If the quantum theory is the output of a functor that has the causal set as its domain, then causality is build into the theory. Even if causality is not the preferred set of conditions on evolution (one may wish to impose a weaker ordering than the causal set, for example a non-transitive order), the same method can be used to check that these conditions are preserved in the quantum theory.

\section{Conclusions}

In this paper, we used the functor Past $: \mathcal{C} \rightarrow$ Set to transform from an external (outside the universe) viewpoint of causality to the internal, finitetime, viewpoint provided by the components of Past at each event. The result provides an algebraic description of causality and great possibilities for generalisations to diverse types of causal quantum theories, some of which we outlined above. Conclusions about the form of quantum evolution that causality permits can be reached in this way. However, note that this would

\footnotetext{
${ }^{12}$ I need to thank Eli Hawkins for this observation.
} 
be considered a kinematical restriction since the causal set on which the evolving set is defined is fixed.

Past is the simplest evolving set, since it only uses the causal set and no additional spatial or field information. It does, however, serve as a good example in introducing evolving sets, the Heyting algebra of evolving powersets, and the non-standard evolving complement. At the classical level (from the perspective of a causal set approach), the technical advantage of Past is that it transforms the partially ordered set of causal relations into a lattice and suggests significant improvements in the description of the causal structure without the use of a spacetime. The actual implementation in the quantum theory will be reported in future work.

Further, it is possible to construct a framework for a (classical) causal cosmological theory by requiring that every physical observable corresponds to an observation made by an observer inside the universe, represented by an event or a collection of events in the set of causal relations of that universe. Such "internal" observables, when referring to events in the observer's causal past, are subfunctors of Past. For example, in the $1+1$ causal histories of Abjorn, Loll and others [16], a physical observable may be events that have spacetime valence $n$ (the number of ingoing and outgoing causal relations to such an event is $n$ ). Written as a varying set, this observable will be the $n$-valent events that have occured at $p$ and is a subfunctor of Past. Such internal causal observables obey a Heyting algebra.

We close by noting that sieves can be used to specify time. This is an algebraic alternative to time expressed as $t \in \mathbf{R}$. In work currently in progress, we investigate projection operators which are time-dependent in the sense that their eigenvalues belong to a larger set than the standard 1 and 0 ; in fact they correspond to sieves.

\section{Acknowledgments}

I am very grateful to Chris Isham for introductory discussions on varying sets and his very useful suggestions and criticisms on the present work. Detailed comments on the first draft from Eli Hawkins, Carlo Rovelli, Lee Smolin and

\footnotetext{
13 After the work in this paper was completed, the paper of Bombelli and Meyer 15] came to our attention. From a given causal set, they construct quantities that contain the same events as Past and $\neg$ Past.
} 
Adam Ritz have made this paper much clearer than it originally was. I am thankful to Sameer Gupta, David Meyer and Roger Penrose for suggestions on using the complement to define a black hole. Thanks are due to John Baez, John Barrett and Alex Heller and particularly to Bas van Fraasen for the first discussions on intuitionistic logic.

This work was supported by NSF grants PHY/9514240 and PHY/9423950 to the Pennsylvania State University and a gift from the Jesse Phillips Foundation.

\section{Appendix: Boolean vs. intuitionistic logic}

This appendix is an account of the basics of Boolean and intuitionistic logic and their relation to the Boolean and Heyting algebras.

The boolean algebra obeyed by sets means that when a physical theory is ultimately built on set-theoretic foundations (which is almost universally the case), the underlying logic is boolean. Loosely speaking, an observer in such a theory will make statements which obey boolean logic. For a theory based on evolving sets, which we propose here, the Heyting algebra of evolving sets indicates that the underlying logic is intuitionistic. For completeness, and because we would like to point the physicist reader to a mathematical literature possibly of use in issues of time evolution in physics, this appendix is a review of both boolean and intuitionistic logic.

From the perspective of a physicist, there is little difference between the algebraic operations and the corresponding logical ones (propositional calculus). Practically, it simply involves "reading" the operations as propositions rather than sets. For example, each proposition $x$ in the logical operations that follow may be replaced by " $a \in A$ " for some subset $A$ of $X$ which we used in section 8 . The algebraic operations of union, intersection, implication, and complement then become the logical connectives OR, AND, IMPLIES and NOT.

Most of the interpretational discussion has already been carried out in section 8. We now simply present the two logical systems. In the intuitionistic case, we include in the presentation some of the historical reasons for the introduction of this type of logic into mathematics. 


\subsection{Boolean logic}

To have either an algebra or a propositional calculus we first need a lattice, as explained in section 8. A lattice is first of all a partial order, so we partial order propositions by $\leq$. The proposition $x \leq y$ is to be read as "if $x$ is true, then $y$ is true".

For a pair of propositions $x$ and $y$, the four boolean logical operations OR, AND, IMPLIES and NOT produce four new propositions. Any further statements made (sentences) are constructed by combinations of these basic operations. They are in obvious correspondence with the four boolean algebra operations.

- OR (union): $(x \vee y)$ is true when either $x$ or $y$ are true.

- AND (intersection): $(x \wedge y)$ is true when both $x$ and $y$ are true.

- IMPLIES (implication): $(x \Rightarrow y)$ is true when, if $x$ is true, then $y$ is also true.

- NOT (complement): The proposition $\neg x$ is true whenever $x$ is false.

We can tabulate the boolean operations:

\begin{tabular}{|c|c||c|c|c|c|}
\hline$x$ & $y$ & $x \vee y$ & $x \wedge y$ & $x \Rightarrow y$ & $\neg x$ \\
\hline \hline 1 & 1 & 1 & 1 & 1 & 0 \\
\hline 1 & 0 & 1 & 0 & 0 & 0 \\
\hline 0 & 1 & 1 & 0 & 1 & 1 \\
\hline 0 & 0 & 0 & 0 & 1 & 1 \\
\hline
\end{tabular}

Note, first, that $\Rightarrow$ as defined above means that

$$
(x \Rightarrow y)=\neg x \vee y,
$$

that is " $x$ implies $y$ " is equivalent to "either $x$ is false or $y$ is true", which with little thinking we can believe (or use the table above).

Second, in a boolean algebra, every proposition $x$ has a negation $\neg x$ which satisfies

$$
x \wedge \neg x=0 \quad \text { and } \quad x \vee \neg x=1,
$$

where 0 means false and 1 means true. As a result, not not $x$ is the same as $x$,

$$
\neg \neg x=x .
$$




\subsection{Intuitionistic logic}

We think it is useful to present intuitionistic logic with reference to the motivation of the mathematicians who invented it. This is standard material and we freely quote from the introduction of [8] and from [17].

Intuitionistic logic and the mathematics based on it originated with Brouwer's work on the foundations of mathematics at the beginning of this century [18]. He insisted that all proofs be constructive. This means that he did not allow proof by contradiction and hence he excluded the classical (boolean) "for all $x$, either $x$, or not $x "$. Intuitionism is a form of constructive mathematics. The classical (boolean) mathematician believes that every mathematical statement $x$ is true or false, whether or not he has the proof for it. The constructive mathematician does not consider $x$ to be true or false unless he can either prove it or disprove it. That is, $x$ may be true tomorrow, or false tomorrow.

To quote Brouwer, "the belief in the universal validity of the excluded middle in mathematics is considered by the intuitionists as a phenomenon in the history of civilization of the same kind as the former belief in the rationality of $\pi$, or the rotation of the firmament about the earth".

Brouwer's approach was not formal or axiomatic, but subsequently Heyting and others introduced formal systems of intuitionistic logic, weaker than classical logic. Heyting first formalised the basic axioms of intuitionism which, usually detached from Brouwer's extreme position, has turned out to be useful for mathematics beyond the original context.

As in the Boolean case, we start with a lattice of propositions, ordered by $\leq$. The four operations work as follows:

- OR and AND are the same as in the boolean case.

- IMPLIES: The statement $(x \Rightarrow y)$ means that $y$ holds under the assumption that $x$ holds, namely, we show $(x \Rightarrow y)$ by deriving $y$ from the hypothesis $x$. (Note that there is no causal implication in $\Rightarrow$, there is no sense of $y$ causally following $x$.) $(x \Rightarrow y)$ is characterized by

$$
z \leq(x \Rightarrow y) \quad \text { if and only if } \quad z \wedge x \leq y,
$$

that is, $(x \Rightarrow y)$, namely the condition $(\sqrt{78})$, is the union of all $z$ that 
satisfy $z \wedge x \leq y$.t4

Note that the constructive interpretation of $(x \Rightarrow y)$ is weaker than the boolean one where $(x \Rightarrow y)=\neg x \vee y$. This leads to the modified intuitionistic negation.

- NOT: The statement $\neg x$ means that $(x \Rightarrow z)$, where $z$ is a contradiction. Usually a contradiction is denoted 0 , so

$$
\neg x=(x \Rightarrow 0) .
$$

that is, we have "not $x$ " when $x$ leads to a contradiction.

From the definition (78) of $\Rightarrow$, we get that

$$
y \leq \neg x \quad \text { if and only if } \quad y \wedge x=0,
$$

namely, $\neg x$ is the union of all propositions $y$ which have nothing in common with $x$. As a result $\neg \neg x$ needs not equal $x$. Also, although $x \wedge \neg x=0$, it may not be the case that $x \vee \neg x=1$.

In short, intuitionistic logic, or, equivalently, a Heyting algebra, is particularly suitable in a theory with time evolution, when we are concerned with physical statements which become true at a certain time stage and stay true afterwards.

\footnotetext{
${ }^{14}$ In the open set example (footnote 8$),(U \Rightarrow V)$ is the union of all open sets $W_{i}$ whose intersection with $U$ is included in $V$, i.e. $(U \Rightarrow V)=\bigcup_{i} W_{i}$ where $W_{i} \cap U \subset V$.
} 


\section{References}

[1] Markopoulou F and Smolin L, 1997, "Causal evolution of spin networks", Nucl Phys B508 409.

[2] Bombelli L, Lee J, Meyer D and Sorkin R, 1987, "Space-time as a causal set", Phys Rev Lett $\mathbf{5 9}$ 521;

Sorkin R, 1990, "Space-time and causal sets" in Proc. of SILARG VII Conf., Cocoyoc, Mexico;

Meyer D A, 1988, The Dimension of Causal Sets, PhD Thesis, Massachussets Institute of Technology.

[3] Bombelli L and Meyer D, 1989, "The origin of Lorentzian geometry", Phys Lett A 141226.

[4] Markopoulou F, 1997, "Dual formulation of spin network evolution", grqc/9704013.

[5] Markopoulou F and Smolin L, 1998, "Quantum geometry with intrinsic local causality", Phys Rev D 58, 084032.

[6] Isham C J, 1997, "Topos theory and consistent histories: the internal logic of the set of all consistent sets", Int J Theor Phys $\mathbf{3 6} 785$.

[7] Isham C J and Butterfield J, 1998, "A topos perspective on the KochenSpecker theorem. 1. Mathematical developments", quant-ph/9808067, "2. Conceptual aspects and classical analogues", quant-ph/9808067.

[8] Mac Lane S and Moerdijk I (1992) Sheaves in Geometry and Logic: A First Introduction to Topos Theory, (London: Springer-Verlag).

[9] Goldblatt R, 1984, Topoi, the categorial analysis of logic (Amsterdam: NorthHolland).

[10] Jacobson T, 1995, "Thermodynamics of spacetime: the Einstein equation of state", Phys Rev Lett $\mathbf{7 5} 1260$.

[11] Sorkin R, 1991, "A finitary substitute for continuous topology", Int J Theor Phys 30923.

[12] Borissov R and Gupta S, 1998, "Propagating spin modes in canonical quantum gravity", gr-qc/9810024. 
[13] Penrose R, 1971, "Theory of quantised directions" in Quantum theory and beyond, ed Bastin T (Cambridge: Cambridge University Press).

[14] Rovelli C and Smolin L, 1995, "Discreteness of area and volume in quantum gravity", Nucl Phys B 593; 734.

For a review, see Rovelli C, 1997, "Loop quantum gravity", gr-qc/9710008.

[15] Bombelli L and Meyer D, 1989, Phys Lett A 141226.

[16] J Ambjorn and R Loll, 1998, "Non-perturbative Lorentzian Quantum Gravity and Topology Change", hep-th/9805108; J Ambjorn, J L Nielsen, J Rolf and R Loll, 1998, "Euclidean and Lorentzian Quantum Gravity: Lessons from 2 dimensions", hep-th/9806241.

[17] Bridges D S, Douglas S and Richman F, 1987, Varieties of constructive mathematics (New York: Cambridge University Press).

[18] Brouwer's Cambridge Lectures on Intuitionism, 1981, ed VanDalen D (Cambridge University Press); 